\title{
Measurement verification of estimation method for time errors in a time-interleaved A/D converter system
}

\author{
Jonas Elbornsson \\ Kalle Folkesson,Jan-Erik Eklund \\ Division of Communication Systems \\ Department of Electrical Engineering \\ Linköpings universitet, SE-581 83 Linköping, Sweden \\ WWW: http://www. comsys.isy.liu.se \\ Email: jonas@isy.liu.se
}

5th November 2001

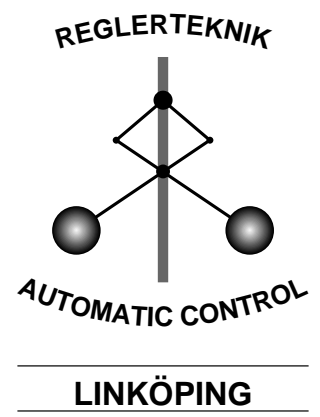

Report No.: LiTH-ISY-R-2402

Submitted to ISCAS'02

Technical reports from the Communication Systems group in Linköping are available by anonymous ftp at the address ftp.control.isy.liu.se. This report is contained in the file 2402 .pdf. 


\begin{abstract}
A previously presented method for estimation of time errors in timeinterleaved A/D converter systems is here verified on measurements from a dual A/D converters system. The advantage of this estimation method, compared to other methods, is that it does not require any knowledge about the input signal. The estimation is most accurate for slowly varying input signals but the signal quality is improved even when the estimation is done for a sinusoidal signal close to the Nyquist frequency.
\end{abstract}

Keywords: sampling, A/D conversion, estimation, measurement 


\section{MEASUREMENT VERIFICATION OF ESTIMATION METHOD FOR TIME ERRORS IN A TIME-INTERLEAVED A/D CONVERTER SYSTEM}

\author{
J. Elbornsson, K. Folkesson \\ Linköping University \\ Department of Electrical Engineering \\ \{jonas,kalfo\}@ isy.liu.se
}

\author{
J.-E. Eklund \\ Ericsson Microelectronics AB \\ jan-erik.eklund@mic.ericsson.se
}

\begin{abstract}
A previously presented method for estimation of time errors in time-interleaved A/D converter systems is here verified on measurements from a dual A/D converters system. The advantage of this estimation method, compared to other methods, is that it does not require any knowledge about the input signal. The estimation is most accurate for slowly varying input signals but the signal quality is improved even when the estimation is done for a sinusoidal signal close to the Nyquist frequency.
\end{abstract}

\section{INTRODUCTION}

Many digital signal processing applications, such as radio base stations or VDSL modems, require ADCs with very high sample rate and very high accuracy. To achieve high enough sample rates, an array of $M$ ADCs, interleaved in time, can be used. Each ADC should work at $1 / M$ th of the desired sample rate $[1,2]$, see Figure 1 . Three kinds of mismatch errors are introduced by the interleaved structure:

- Time errors

- Offset difference

- Gain difference

We consider only the time errors in this paper. The time errors are assumed to be static, so that the error is the same in the same ADC from one cycle to the next.

Methods for estimation of timing errors have been presented in for instance [3] and [4] but those methods require a known calibration signal. Calibration of ADCs is time-consuming and expensive. Therefore a lot of costs can be saved if the errors in the ADC can be automatically estimated and compensated for at run-time.

We will in this paper review an estimation method for time errors in interleaved ADCs, [5, 6]. The estimation method does not require any prior knowledge about the input signal, except that it should be band limited to the Nyquist frequency. The estimated time errors are then used for correcting the output signal. In order to show the quality of the estimates, we correct the data by interpolation in the frequency domain. The results in $[5,6]$ are based on simulations. The estimation method is here verified on measurements from a dual A/D converters system.

\section{THEORY}

In this section the estimation and compensation algorithms are briefly described. A more complete description of the estimation algorithm is given in $[5,6]$.

\subsection{Notation}

The analog input signal is denoted $u(t) . T_{s}$ denotes the nominal sampling interval. $M$ is the number of ADCs. The time error for the $i$ th $\mathrm{ADC}$ is denoted $t_{i}$. The output from the $i$ th ADC

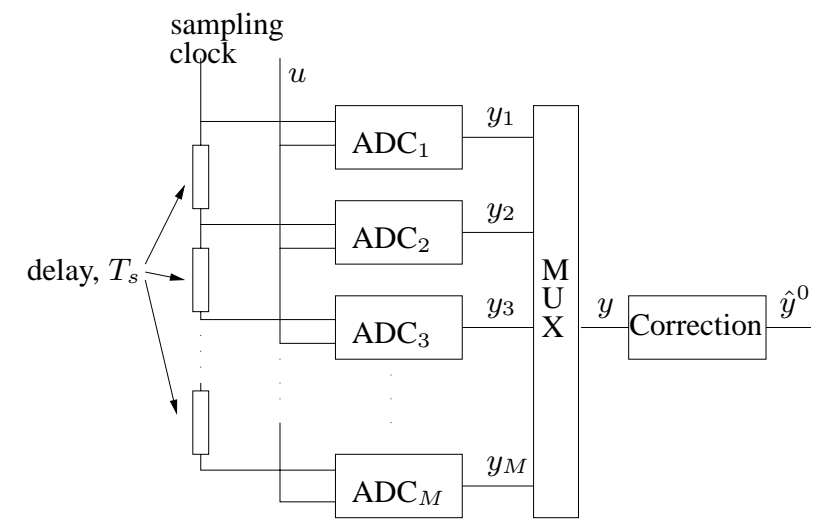

Fig. 1. $M$ parallel ADCs with the same master clock.

is denoted $y_{i}[k]$, where $k$ is the $k$ th sample from that ADC. $N$ denotes the number of samples from each ADC. $y[k]$ denotes the non-uniformly sampled signal and $\hat{y}^{0}[k]$ denotes the estimated uniformly sampled signal.

\subsection{Time error estimation method}

The basic idea of the estimation method is that the signal changes more on average if the sampling interval is longer than the nominal sampling interval and vice versa, see Figure 2. We assume, for this estimation algorithm, that the input signal is band limited.

\subsubsection{Estimation algorithm}

A crude estimate of the time errors is first calculated as

$$
\begin{aligned}
& t_{i}^{(0)}=T_{s} \sum_{j=2}^{i}\left(\sqrt{\frac{\hat{R}_{j, j-1}^{N}[0]}{\frac{1}{M} \sum_{i=1}^{M} \hat{R}_{i, i-1}^{N}[0]}}-1\right) \\
& i=2, \ldots, M
\end{aligned}
$$

where $\hat{R}_{i, i-1}^{N}[0]$ is calculated from measured data as

$$
\hat{R}_{i, i-1}^{N}[0]=\frac{1}{N} \sum_{k=1}^{N}\left\{y_{i}[k]-y_{i-1}[k]\right\}^{2}
$$




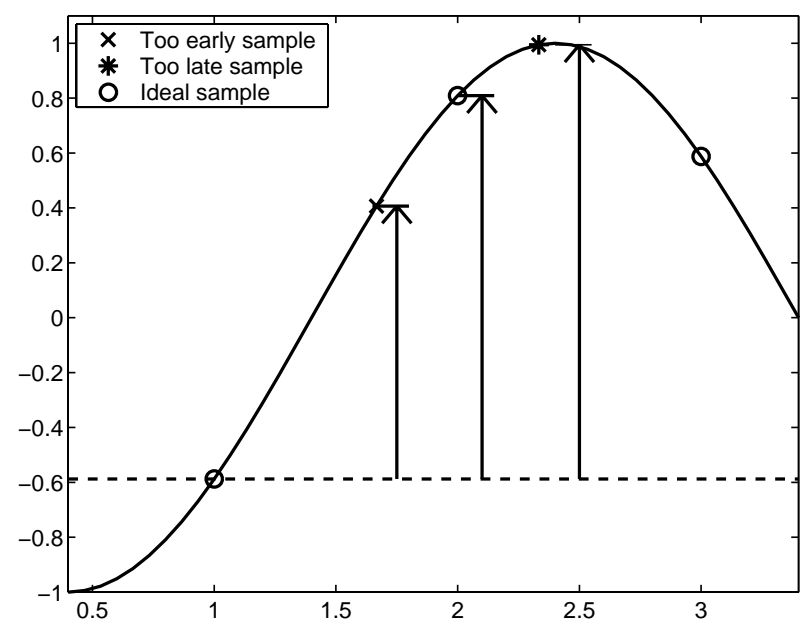

Fig. 2. The basic idea for the timing error estimation method. If the sample is taken too early the average difference between adjacent sample values is smaller, if the sample is taken too late the average difference between adjacent samples is larger.

The time error estimates can then be improved by fixed-point iteration:

$$
\begin{aligned}
& \text { for } i=2, \ldots, M \\
& t_{i}^{(l)}=T_{s} \sum_{j=2}^{i}\left(\sqrt{\frac{1}{\frac{\hat{R}_{j, j-1}^{N}}{a^{(l-1)}} \sum_{i=1}^{M} \hat{R}_{i, i-1}^{N}[0]}}-1\right) \\
& a^{(l)}=M\left(1+\frac{2}{M} \sum_{i=1}^{M}\left(\frac{t_{i}^{(l)}}{T_{s}}\right)^{2}-\frac{2}{M} \sum_{i=1}^{M} \frac{t_{i}^{(l)}}{T_{s}} \frac{t_{i-1}^{(l)}}{T_{s}}\right)
\end{aligned}
$$

\subsection{Correction through interpolation}

When the time errors are estimated, we need to estimate the uniformly sampled signal from the measured non-uniformly sampled signal. The reconstruction is done in the frequency domain [7]. Calculate the DFTs of the $M$ subsequences $y_{i}[k], i=1, \ldots, M$ :

$$
Y_{i}[n]=\operatorname{DFT}\left\{y_{i}[k]\right\}
$$

The DFT of $\hat{y}_{i}^{0}[k]$ can then be calculated from $Y_{i}[n]$ as

$$
\hat{Y}_{i}^{0}[n]=e^{-j \frac{2 \pi n t_{i}}{M N}} Y_{i}[n], n=-N / 2, \ldots, N / 2-1
$$

$\hat{Y}^{0}[n]$ can then be calculated from these $M$ subsequences [8]

$$
\begin{aligned}
& \hat{Y}^{0}[n]=\sum_{i=1}^{M} e^{-j \frac{2 \pi(i-1) n}{M N}} \hat{Y}_{i}^{0}[(n \bmod N)-N / 2] \\
& n=-N M / 2, \ldots, N M / 2-1
\end{aligned}
$$

The estimated uniformly sampled signal is then calculated as

$$
\hat{y}^{0}[k]=\operatorname{IDFT}\left\{\hat{Y}^{0}[n]\right\}
$$

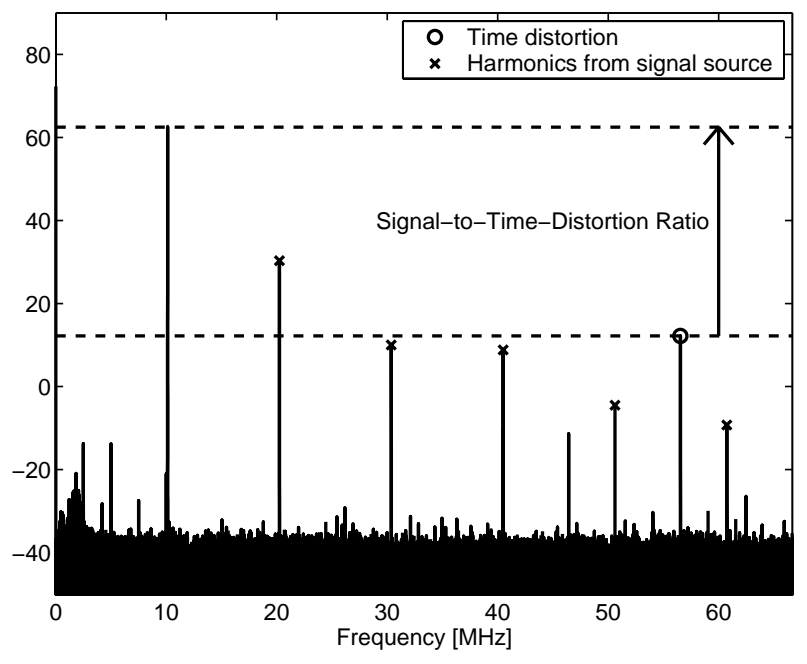

Fig. 3. Definition of the Signal-to-Time-Distortion Ratio

\subsection{Time error distortion}

The frequency synthesizer that is used as signal source typically has high harmonic distortion. However, the posistion of the distortion caused by the time error is, for a dual ADCs system, given by $f_{N}-f_{i n}$, where $f_{N}$ is the Nyquist frequency and $f_{i n}$ is the input signal frequency. This means that we can study only the improvement of the tone caused by the time error without having to bother about the quality of the signal source. The distance between the energy of the signal peak and the energy of the distortion peak caused by the time error is measured, see Figure 3 . We will denote this measure signal-to-time-distortion ratio (STDR).

We will here calculate the signal quality as a function of the size of the time error for a sinusoidal input, $u(t)=\sin (\omega t)$. We assume, for simplicity in the calculations, that $M=2$ and that $\omega=\frac{2 \pi a}{N M}$ where $a$ is an integer. This means that $y[k]$ is formed from the two subsequences

$$
\begin{aligned}
& y_{1}[k]=\sin \left(\frac{2 \pi a}{N M} 2 k\right) \\
& y_{2}[k]=\sin \left(\frac{2 \pi a}{N M}\left(2 k+1+t_{2}\right)\right)
\end{aligned}
$$

The DFT of $y[k]$ is

$$
Y[n]= \begin{cases}\frac{N}{2 j}\left(1+e^{j \frac{\pi a}{N} t_{2}}\right) & \text { if } n=a \\ \frac{N}{2 j}\left(-1+e^{-j \frac{\pi a}{N} t_{2}}\right) & \text { if } n=N-a \\ \frac{N}{2 j}\left(-1+e^{-j \frac{\pi a}{N} t_{2}}\right) & \text { if } n=N+a \\ \frac{N}{2 j}\left(1+e^{j \frac{\pi a}{N} t_{2}}\right) & \text { if } n=2 N-a \\ 0 & \text { otherwise }\end{cases}
$$

The signal-to-time-distortion ratio is then

$$
S T D R=20 \log \left(\left|\frac{1+e^{j \frac{\pi a}{N} t_{2}}}{1-e^{-j \frac{\pi a}{N} t_{2}}}\right|\right)
$$

A Taylor expansion of (9) gives

$$
S T D R \approx 20 \log 2-20 \log \left(\frac{2 \pi a t_{2}}{2 N}\right)
$$

This means that the signal-to-time-distortion ratio is decreased approximately $6 d B$ per octave, see Figure 6 . 


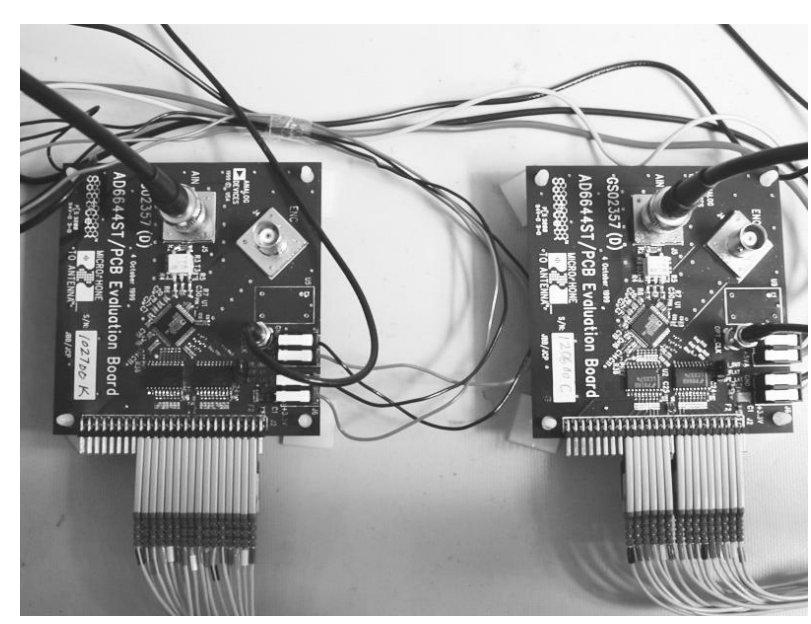

(a)

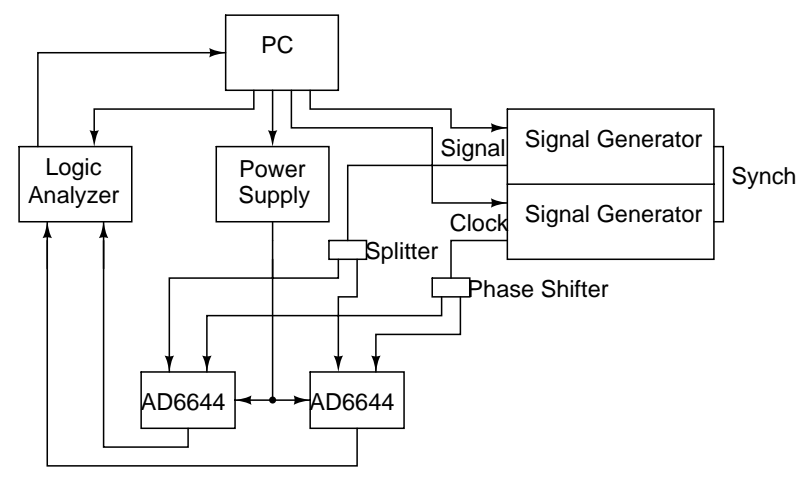

(b)

Fig. 4. (a) Two AD6644 evaluation boards. (b) Measurement setup: Clock signal and input signal are generated from the two signal generators. Time interleaving is achieved by inverting the clock signal to one ADC. The output signal is collected in the logic analyzer and the signal processing is done in MATLAB.

\section{MEASUREMENTS}

In this section, the measurement verification of the algorithm described in Section 2, is presented.

\subsection{Measurement setup}

Measurements were done using two AD6644 evaluation boards from Analog Devices [9], with a sampling frequency of $66.6 \mathrm{MHz}$ each, see Figure 4(a). A signal generator was used as clock signal and a differential pulse splitter was used to create two clock signals with opposite phase, thereby doubling the sampling frequency. A signal generator was used as input signal, see Figure 4(b).

\subsection{Data acquisition}

The measured data were collected from the logic analyzer and MATLAB was used for signal processing. To look only at time errors, offset and gain errors need to be eliminated. This can be done by various mean value calculations and is not a subject of this paper.
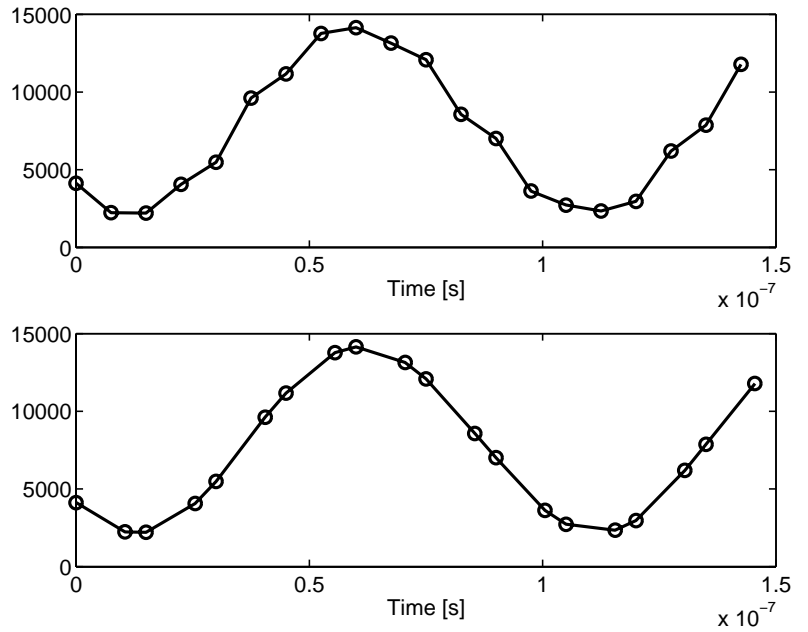

Fig. 5. Measured signal with $0.4 T_{s}$ clock delay on one ADC. Upper plot: signal sampled with non-uniform sample interval but interpreted with uniform sample interval. Lower plot: the same signal, but interpreted with a non-uniform sample interval.

The time error estimation algorithm was evaluated with sinusoidal input signals. Nine batches of data at different frequencies between $5 \mathrm{MHz}$ and $60 \mathrm{MHz}$ were collected. For each input signal frequency, 65536 samples per channel were collected.

\subsection{Evaluation}

The estimation algorithm does not utilize any prior information about the input signal. Therefore, the fact that we know that the input signal is sinusoidal is not used in the algorithm and does not influence the performance of the estimation algorithm. The reason why we have chosen a sinusoidal input signal is that it is easy to generate and that there exist good signal quality measures for sinusoidal signals. We use the signal-to-time-distortion ratio for evaluation of the signal quality, see Section 2.4.

\subsubsection{With extra delay for visibility}

In order to visualize the effect of the time error, the clock to one of the ADCs was delayed $3.2 n s \approx 0.4 T_{s}$ by adding a $0.5 \mathrm{~m}$ coaxial cable. Figure 5 shows the measured signal with and without compensation for the timing error. The compensation was here done by moving the sampling instances with the estimated time error. The input signal frequency was here $10 \mathrm{MHz}$.

\subsubsection{Normal operation}

The time error is here much smaller, $0.17 n s \approx 0.02 T_{s}$, and can not be seen in the time domain. Instead we study the distortion component in the frequency domain before and after estimation and compensation as described in Section 2. Figure 6 shows the theoretical and measured signal-to-time-distortion ratio without time error compensation. Figure 7(a) shows the improvement of the signal-to-time-distortion ratio after compensation and in Figure 7(b) the signal-to-time-distortion ratio after compensation is shown. The result of the estimation is here shown for three input signals:

- A $10 \mathrm{MHz}$ sinusoidal signal, 131072 samples.

- A 40MHz sinusoidal signal, 131072 samples.

- Sinusoids of nine different frequencies between $5 \mathrm{MHz}$ and $60 \mathrm{MHz}$ concatenated into one signal, 1179648 samples. 


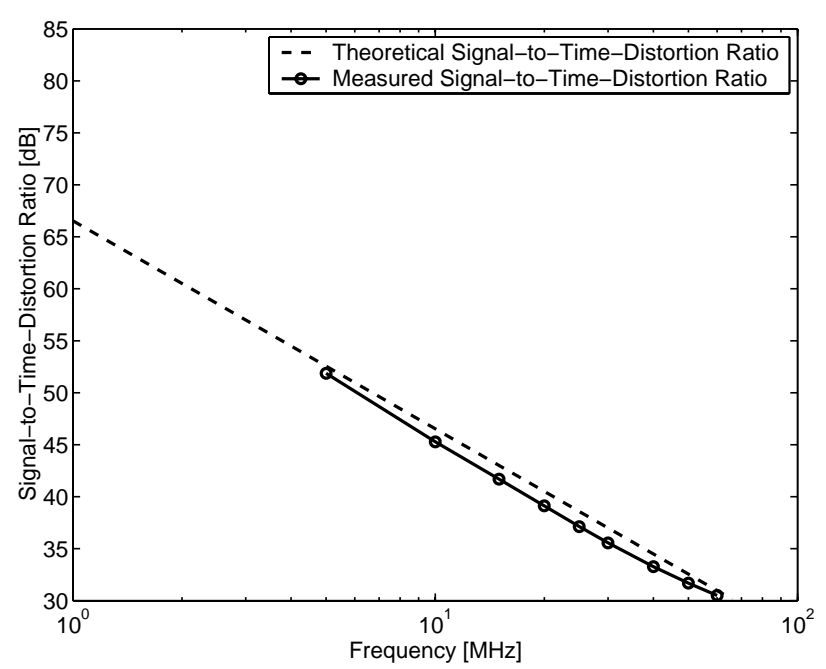

Fig. 6. Comparison between theoretical Signal-to-Time-Distortion Ratio calculated from Equation (9) (dashed line) and measured Signal-to-Time-Distortion Ratio (solid line), without compensation.

\section{CONCLUSIONS}

We have evaluated an estimation method for time errors on measurements from a time-interleaved A/D converter system. The method does not require any knowledge about the input signal. We have done the evaluation with sinusoidal input signals at different frequencies. The knowledge about the input signal is not used in the estimation algorithm. The choice of input signal is motivated by the fact that it is easy to generate sinusoidal signals and that there exist good signal quality measures for them.

We have verified, with many different input signals, that the signal quality is improved after compensation. The measurements show that the improvement is better for lower frequencies and tends to zero near the Nyquist frequency. This result agrees with the theory $[5,6]$.

\section{REFERENCES}

[1] Y-C Jenq, "Digital spectra of nonuniformly sampled signals: A robust sampling time offset estimation algorithm for ultra high-speed waveform digitizers using interleaving," IEEE Transactions on Instrumentation and Measurement, vol. 39, no. 1, pp. 71-75, February 1990.

[2] W.C. Black and D.A. Hodges, "Time interleaved converter arrays," IEEE Journal of Solid-State Circuits, vol. 15, no. 6, pp. 1022-1029, December 1980.

[3] J.J. Corcoran, "Timing and amplitude error estimation for time-interleaved analog-to-digital converters," US Patent nr. 5,294,926, October 1992.

[4] H. Jin and E.K. Lee, "A digital-background calibration technique for minimizing timing-error effects in time-interleaved ADC's," IEEE Transactions on Circuits and Systems, vol. 47, no. 7, pp. 603-613, July 2000.

[5] J. Elbornsson and J.-E. Eklund, "Blind estimation of timing errors in interleaved AD converters," in Proc. ICASSP 2001. IEEE, 2001, vol. 6, pp. 3913-3916.

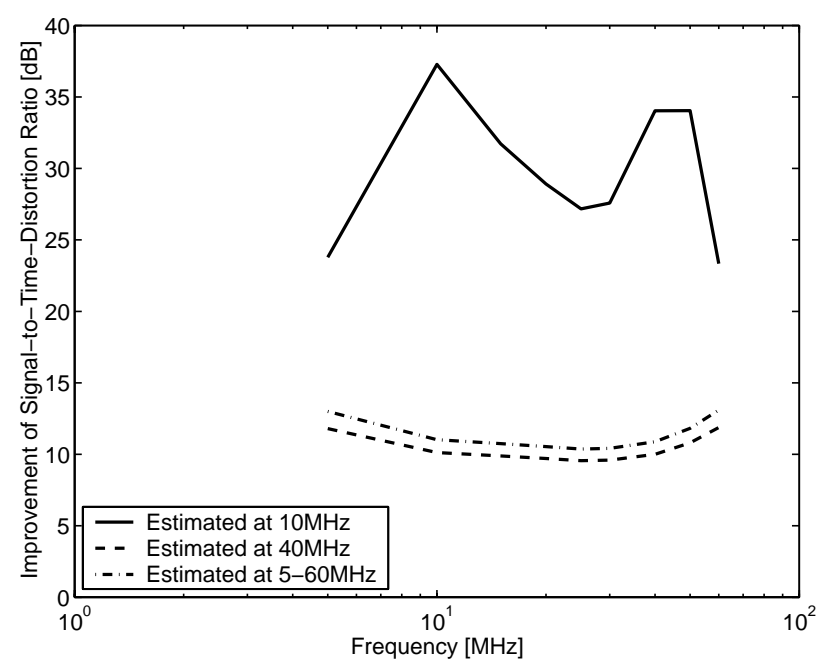

(a)

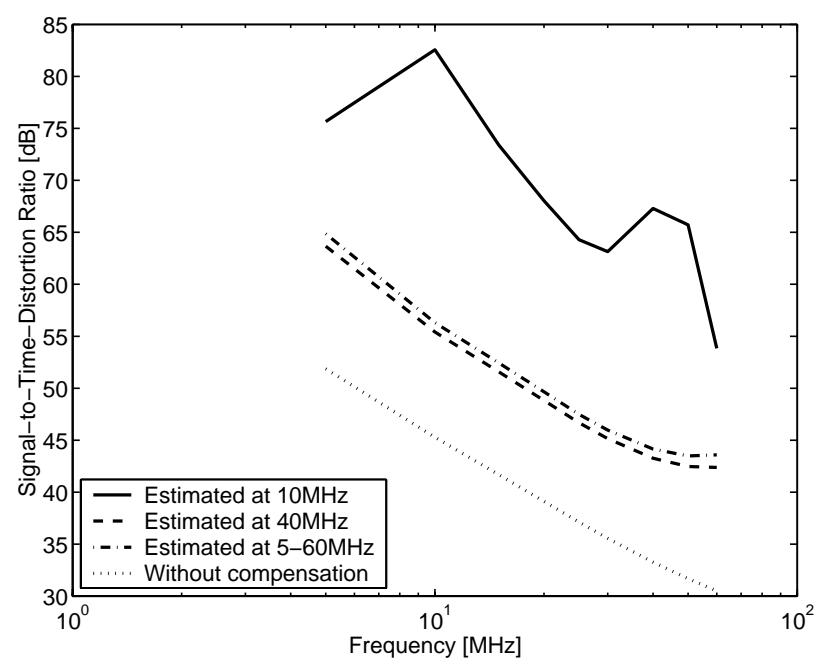

(b)

Fig. 7. (a) Improvement of signal-to-time-distortion ratio after time error compensation. (b) Signal-to-time-distortion ratio after time error compensation. The time error estimation has been done for different input signals: Sinusoidal signal at $10 \mathrm{MHz}$ (solid), Sinusoidal signal at $40 \mathrm{MHz}$ (dashed) and Sinusoidal signals between $5 \mathrm{MHz}$ and $60 \mathrm{MHz}$ concatenated (dash-dotted). The uncompensated curve is shown as reference (dotted).

[6] J. Elbornsson, Equalization of Distortion in A/D Converters, Lic. thesis 883, Department of Electrical Engineering, Linköping University, Linköping, Sweden, April 2001.

[7] A. Papoulis, Signal Analysis, McGraw-Hill, 1977.

[8] F. Gustafsson, L. Ljung, and M. Millnert, Digital Signalbehandling, Studentlitteratur, 2001, in Swedish.

[9] Analog Devices, “AD6644 14-bit, 65 MSPS A/D converter datasheet," 2000. 